\title{
A combination of antibodies against Bm86 and Subolesin inhibits engorgement of Rhipicephalus australis (formerly Rhipicephalus microplus) larvae in vitro
}

Jos J. A. Trentelman ${ }^{{ }^{*}}$ (10, Hendry Teunissen², Jos A. G. M. Kleuskens ${ }^{3}$, Jos van de Crommert ${ }^{4}$, José de la Fuente ${ }^{5,6}$, Joppe W. R. Hovius ${ }^{1}$ and Theo P. M. Schetters ${ }^{7,8}$

\begin{abstract}
Background: Rhipicephalus microplus is a hard tick species that has a high impact on cattle health and production in tropical and subtropical regions. Recently, ribosomal DNA and morphological analysis resulted in the reinstatement of $R$. australis as a separate species from $R$. microplus. Both feed on cattle and can transmit bovine pathogens such as Anaplasma and Babesia species. The current treatment with acaricides is becoming increasingly less effective due to the emergence of resistant tick strains. A promising alternative can be found in the form of anti-tick vaccines. The available commercial vaccines can be used to control tick infestation, but the lack of a knockdown effect (>90\% reduction in tick numbers as seen with effective acaricides) hampers its widespread use, hence higher efficacious vaccines are needed. Instead of searching for new protective antigens, we investigated the efficacy of vaccines that contain more than one (partially) protective antigen. For screening vaccine formulations, a previously developed in vitro feeding assay was used in which $R$. australis larvae are fed sera that were raised against the candidate vaccine antigens. In the present study, the efficacy of the Bm86 midgut antigen and the cytosolic Subolesin (SUB) antigen were evaluated in vitro.
\end{abstract}

Results: Antiserum against recombinant Bm86 (rBm86) partially inhibited larval engorgement, whereas antiserum against recombinant SUB (rSUB) did not have any effect on feeding of larvae. Importantly, when larvae were fed a combination of antiserum against rBm86 and rSUB, a synergistic effect on significantly reducing larval infestations was found. Immunohistochemical analysis revealed that the rBm86 antiserum reacted with gut epithelium of $R$. australis larvae, whereas the antiserum against rSUB stained salivary glands and rectal sac epithelium.

Conclusions: Combining anti-Bm86 and anti-subolesin antibodies synergistically reduced R. australis larval feeding in vitro. Rhipicephalus australis is a one host tick, meaning that the larvae develop to nymphs and subsequently adults on the same host. Hence, this protective effect could be even more pronounced when larvae are used for infestation of vaccinated cattle, as the antibodies could then affect all three developmental stages. This will be tested in future in vivo experiments.

Keywords: Artificial tick feeding, In vitro screening, R. microplus, R. australis, Subolesin, Bm86, Vaccine

\footnotetext{
*Correspondence: j.j.trentelman@amsterdamumc.nl

${ }^{1}$ Center for Experimental and Molecular Medicine, Amsterdam Infection

\& Immunity, Amsterdam UMC, Univ of Amsterdam, Meibergdreef 9,

1105AZ Amsterdam, The Netherlands

Full list of author information is available at the end of the article
} 


\section{Background}

Rhipicephalus microplus is a hard tick that has a major impact on cattle health in tropical and subtropical regions. Tick attachment and feeding on cattle has a direct negative effect on cattle production [1]. Recently, ribosomal DNA and morphological analysis resulted in the reinstatement of $R$. australis as a separate species from $R$. microplus [2]. Next to production loss through feeding, ticks can transmit a range of diseases, including anaplasmosis and babesiosis. It is therefore of great importance to control tick infestations to ensure livestock health, productivity and the livelihood of rural smallholder communities. To date, tick control heavily depends on the use of tick-resistant breeds and treatment of susceptible breeds with acaricides, but tick resistance to these acaricides is becoming problematic [3].

An alternative to acaricide treatment could be vaccination with tick antigens. Early studies have shown that vaccination with crude tick antigen preparations was indeed able to induce antibodies and interfere with feeding and subsequent further development, thereby reducing tick infestation [4]. However, preparation of crude tick extracts is cumbersome and not feasible for the development of a commercial anti-tick vaccine. With the arrival of recombinant protein techniques, single protein antigens could be evaluated for protective activity. This led to the commercial and industrial production of Bm86, a tick midgut antigen first described in 1989 [5] which forms the basis of two commercial anti-tick vaccines (Gavac ${ }^{\mathrm{TM}}$, Heber Biotech; TickGard, Merck Animal Health) $[6,7]$. The efficacy of these vaccines in the field was estimated on average $55 \%$ reduction of the number of engorged adult female $R$. microplus ticks, which hampers its widespread use [6].

After the discovery of $\mathrm{Bm} 86$ and its success as the first recombinant anti-tick vaccine, numerous studies have been performed, identifying multiple tick antigens as reviewed previously $[8,9]$. From these reviewed antigens, Ribosomal protein P0 was shown to have the highest overall efficacy of $96 \%$ [10]. However, as experimental vaccination studies with these antigens showed a maximal reduction of $70 \%$ on the number of engorged female adults, none of these appear to be a vast improvement over the current Bm86-based commercial vaccines nor approaching the efficacy of acaricides. Vaccination with partially purified tick extracts increased protection against $R$. microplus compared to $\mathrm{Bm} 86$ alone, indicating that the effect of Bm86-based vaccines could be increased through the addition of other tick antigens [11]. Hence, it seems more feasible to build on Bm86-based vaccines and increase their anti-tick efficacy by optimizing their formulation.
A more recently described antigen is Subolesin (SUB). It was discovered in 2003 through cDNA Expression Library Immunization of an I. scapularis derived IDE8 embryonic cell line and subsequent Expressed Sequence Tag analysis [12] under the name of 4D8, later renamed Subolesin [13]. Phylogenetic analysis showed that SUB is an orthologue of Akirin [14]. Akirin is involved in the innate immune response of Drosophila melanogaster and is thought to function as a transcription factor in NF- $\mathrm{KB}$ dependent gene expression in insects and mammals [15]. Knock out of the SUB gene in RNAi experiments showed degeneration of salivary glands, midgut and reproductive organs [13]. RNAi of SUB showed a negative effect on tick weight and high reduction in tick progeny after feeding for multiple hard tick species $[13,16]$. It has also been shown that RNAi with SUB reduced $R$. microplus infestation rate, tick weight and oviposition, whereas vaccination only affected $R$. microplus infestation rate and oviposition $[17,18]$.

Currently, vaccination-challenge trials in cattle are being used to evaluate and select $R$. microplus and $R$. australis candidate vaccine antigens. However, these experiments are costly, time consuming and are under limitation due to their negative impact on animal welfare. An in vitro feeding model for $R$. australis is an attractive alternative to evaluate the anti-tick effect of immune sera. There have been multiple reports of the use of in vitro feeding for ticks in literature [19-31]. There are two methods to feed ticks in vitro; capillary feeding and membrane feeding. Using capillary feeding, ticks are fed to repletion by placing capillary tubes over the hypostome [19-21]. This technique is limited to the feeding of semi-engorged adult ticks as the ticks mouthparts need to be large enough to fit the capillary tube and ticks have to be very eager to imbibe blood. Membrane feeding tries to mimic the natural situation where ticks attach to either animal skin or an artificial membrane [22-25]. Depending on the membrane used, membrane feeding can be used for larval and nymphal ticks as well [26-28]. In both systems, ticks are fed naïve bovine blood (defibrinated or supplemented with anti-coagulants) and when in vitro efficacy of tick antigens are tested, specific antibodies or anti-serum was added. It is known that natural tick immunity consists of both humoral and cellular immune components. However, it can be partially transferred by antibodies alone. This led Evin \& Kemp to postulate that an anti-tick vaccine should consist of antigens that can be targeted by antibodies and the formation of the antibodyantigen complex should disrupt vector biology [32-34]. As defibrinated blood comes from naive animals and the specific anti-serum does not contain immune cells, any observed anti-tick effect in vitro would, therefore, be highly antibody mediated. Consequently, any observed 
anti-tick effect with in vitro feeding could be an underestimation of an anti-tick effect in vivo as cellular and humoral immune components interplay in vivo. In vitro feeding of larvae with small hypostomes as $R$. microplus through an artificial membrane proved to be highly challenging. Only recently we described the development of an in vitro feeding system for $R$. australis larvae that can be used to evaluate the inhibitory activity of antisera against tick antigens [35]. Here we evaluated the in vitro effect of antisera against Bm86, antisera against SUB, and combinations thereof on engorgement of $R$. australis larvae.

\section{Methods}

\section{Tick larvae}

Tick larvae were obtained from a colony of $R$. australis that was routinely passaged on Holstein calves (Merck Animal Health Innovation GmbH, Schwabenheim, Germany). Fully engorged female ticks were collected from the calves and allowed to oviposit in Petri dishes. The resulting egg-masses were collected in laboratory tubes and allowed to hatch at $22{ }^{\circ} \mathrm{C}$ and $90 \%$ humidity. Four to six-week-old $R$. microplus larvae were used in the feeding experiments.

\section{Tick naïve bovine serum}

For the production of normal serum, blood from healthy tick naïve Holstein Friesian cattle was collected in BD Vacutainer ${ }^{\circledR}$ Plus plastic serum tubes. Blood was allowed to clot for $1 \mathrm{~h}$ at $37^{\circ} \mathrm{C}$, centrifuged for $15 \mathrm{~min}$ at $1000 \times g$, serum was removed and stored at $-20^{\circ} \mathrm{C}$.

\section{Antigen production, vaccination and serum collection}

Recombinant Bm86 was produced in the Baculovirus expression system as described before [35]. Recombinant SUB was produced in E. coli, inclusion bodies denatured with $6 \mathrm{M}$ Ureum buffer and SUB was subsequently purified using a HIS-trap column (Profinia IMAC cartridge, Biorad, California, USA) and dialysed against $50 \mathrm{mM}$ MES buffer (Fig. 1). Production of recombinant protein was confirmed by western blot with rabbit anti-rBm86 (Pichia pastoris-produced) antiserum (1:100) and with a mouse monoclonal antibody (MSD Animal Health, Boxmeer, Netherlands) against poly-histidine ( $\mathrm{His}_{6}$-tail) for isolated rSUB-HIS 6 . Antigen-specific bovine serum was produced as follows: five Friesian Holstein cattle were subcutaneously vaccinated 2 times at a 3-week interval in the neck region with either Bm86 or SUB in water in oil adjuvant (Montanide ISA 50V2, Seppic, Paris, France).

Two weeks after the last vaccination with each antigen, blood was collected for serum production. Serum was pooled before feeding and antibody reactivity was quantified through ELISA (see below).

\section{Bm86 ELISA}

Anti-Bm86 bovine serum titers were tested in a sandwich ELISA. In short, purified IgG from rabbit anti-rBm86 (Pichia pastoris-produced) antiserum $(5 \mu \mathrm{g} / \mathrm{ml}$ in bicarbonate/carbonate coating buffer) was coated overnight on a Greiner F ELISA plate at room temperature. The wells were subsequently blocked for $1 \mathrm{~h}$ with $200 \mu \mathrm{l} /$ well $1 \% \mathrm{w} / \mathrm{v}$ bovine serum albumin (BSA) in $0.04 \mathrm{M}$ isotonic PBS at $37{ }^{\circ} \mathrm{C}$. Next, Baculovirus-produced rBm86 was added to the plate $(0.12 \mu \mathrm{g} / \mathrm{ml}$ in $1 \% \mathrm{w} / \mathrm{v}$ BSA in EIAtween 80 buffer, $100 \mu \mathrm{l} /$ well) and left to incubate for $2 \mathrm{~h}$ at $37^{\circ} \mathrm{C}$. Vaccinated cattle serum was diluted [in $1 \% \mathrm{w} / \mathrm{v}$ BSA in GLD/1 buffer supplemented with $10 \%(\mathrm{v} / \mathrm{v})$ naïve dog serum] and $100 \mu \mathrm{l} /$ well subsequently added to the plate for $1 \mathrm{~h}$ incubation at $37{ }^{\circ} \mathrm{C}$. Goat anti-bovine IgGHRP (Jackson ImmunoResearch Inc., Westgrove, USA) was 2500 times diluted in $1 \% \mathrm{w} / \mathrm{v}$ BSA in EIA-tween 80 buffer and $100 \mu \mathrm{l} /$ well added to incubate for $1 \mathrm{~h}$ at $37^{\circ} \mathrm{C}$. Finally, $100 \mu \mathrm{l} /$ well substrate $(185 \mu \mathrm{l}$ TMB and $1 \mathrm{ml}$ UPbuffer in $10 \mathrm{ml}$ water for injection) was added and left to incubate for $15 \mathrm{~min}$ in the dark at room temperature. The reaction was stopped with $50 \mu \mathrm{l} /$ well $4 \mathrm{~N} \mathrm{H}_{2} \mathrm{SO}_{4}$ and OD was measured at $450 \mathrm{~nm}$. Antibody titres were calculated as end point titres (Cut off is Bmin*2) using the ABend Vertical CBA v2.29 software package (MSD animal Health, Boxmeer, Netherlands).

\section{SUB ELISA}

Antibody titres against $\mathrm{rSUB}^{-\mathrm{HIS}_{6}}$ recombinant antigen were determined using a sandwich ELISA. Briefly, Greiner F ELISA-plates were coated overnight with a mouse monoclonal antibody (MSD Animal Health, Boxmeer, Netherlands) against poly-histidine ( $\mathrm{His}_{6}$-tail). Plates were washed and blocked with $1 \% \mathrm{w} / \mathrm{v}$ BSA in 0.04 $\mathrm{M}$ isotonic PBS and a standard amount of rSUB-His antigen in EIA-tween 80 was added to the plates. After incubation three-fold serial dilutions of serum samples in $1 \% \mathrm{w} / \mathrm{v}$ BSA and $10 \% \mathrm{v} / \mathrm{v}$ naïve dog serum in GLD1 buffer was added. Next, total bound immunoglobulin antibodies were detected by incubation with a secondary antibody goat-anti-bovine IgG conjugated with peroxidase. Finally, $100 \mu \mathrm{l} /$ well substrate $(185 \mu \mathrm{l} \mathrm{TMB}$ and $1 \mathrm{ml}$ UP-buffer in $10 \mathrm{ml}$ water for injection) was added and left to incubate for $15 \mathrm{~min}$ in the dark at room temperature. The reaction was stopped with $50 \mu \mathrm{l} /$ well $4 \mathrm{~N}_{2} \mathrm{SO}_{4}$ and OD was measured at $450 \mathrm{~nm}$. Antibody titres were calculated as end point titres (Cut off is Bmin*2) using the ABend Vertical CBA v2.29 software package (MSD Animal Health).

\section{Immunohistochemistry}

Unfed $R$. australis larvae were fixed with $4 \% \mathrm{v} / \mathrm{v}$ formaldehyde, later dehydrated for $30 \mathrm{~min}$ at each of the 


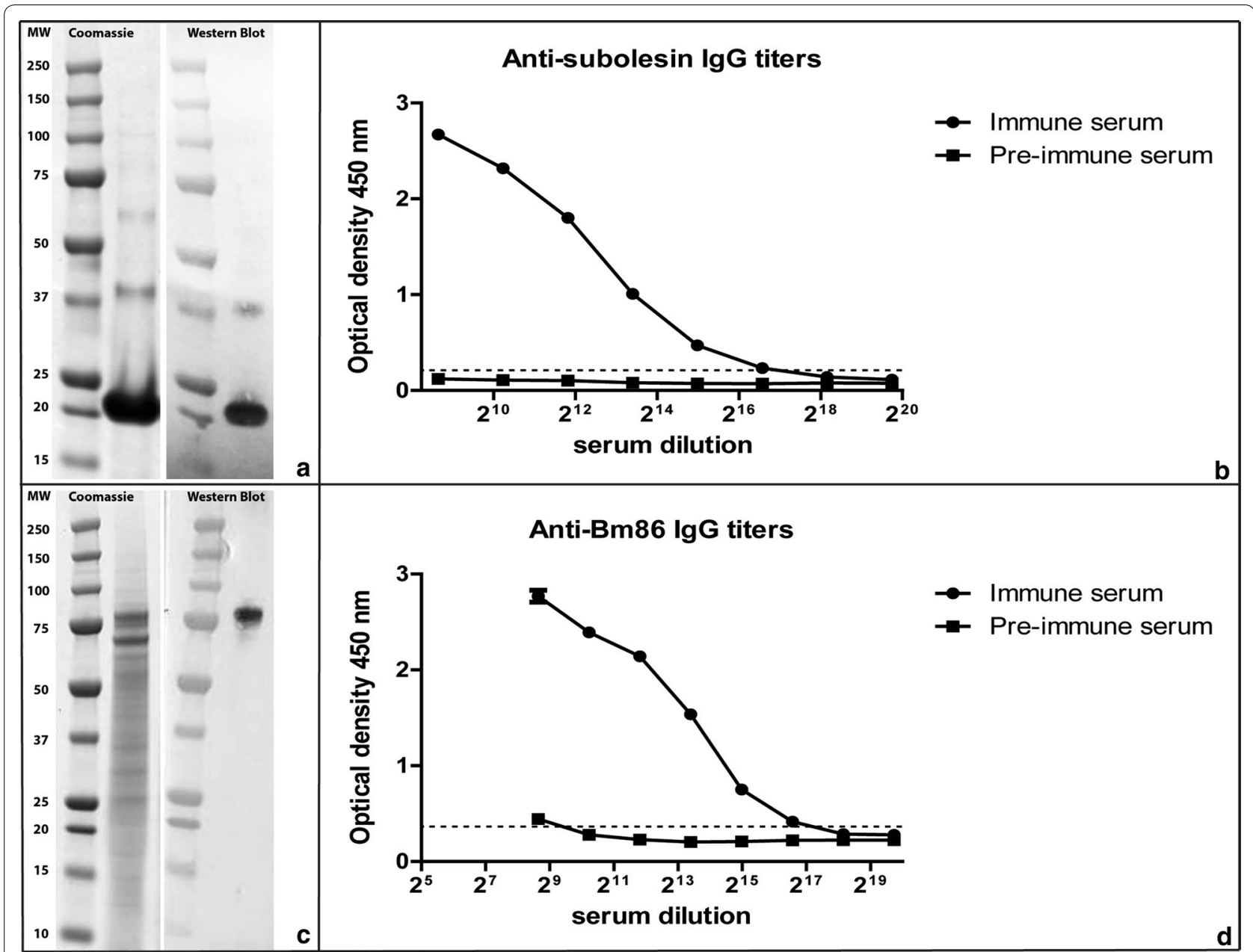

Fig. 1 Recombinant Bm86 and subolesin and determination of antigen specific antibody titers. a Coomassie staining (left) and Western blot using anti-HIS mouse lgG (right) of purified subolesin (SUB) using a 4-20\% Bis-Tris gel. b Anti-SUB antibody titration by sandwich ELISA; recombinant SUB was captured with anti-HIS mouse lgG and pooled SUB vaccinated cow serum was diluted to calculate endpoint titers. End-point titer cut-off (Bmin*2) is indicated by dashed line. c Coomassie staining (left) and Western blot using Bm86 (P. pastoris-produced) specific rabbit lgG (right) of recombinant Bm86. d Anti-Bm86 antibody titration by sandwich ELISA adapted from Trentelman et al. [35]. Bm86 (baculovirus produced) was captured with Bm86 (P. pastoris-produced) specific rabbit lgG and pooled SUB vaccinated cow serum was diluted to calculate endpoint titers. End point titer cut-off (Bmin*2) is indicated by dashed line

following ethanol concentrations, $70 \%, 80 \%, 90 \%$ and $100 \% \mathrm{v} / \mathrm{v}$, routinely embedded in paraffin wax and $3-5$ $\mu \mathrm{m}$ sections were made. Hematoxylin and eosin staining was performed following routine histological procedures. Sections used for labeling with anti-rSUB antibodies were blocked with $1 \% \mathrm{w} / \mathrm{v}$ BSA, incubated with anti-rSUB rabbit serum (1:400). Naïve rabbit serum (1:400) was used as a control. Bm86 antigen detection was performed by labeling with anti-rBm86 antibodies after pre-incubation of the thin sections with proteinase $\mathrm{K}$ for $30 \mathrm{~min}$. Slides were subsequently blocked with $1 \% \mathrm{w} / \mathrm{v}$ BSA, incubated with anti-rBm86 rabbit serum (1:400). As a control naïve rabbit serum (1:400) was used.

\section{Artificial tick feeding}

The feeding units were used as described before [35]. Feeding membranes were made from baudruche membranes of less than $30 \mu \mathrm{m}$ thickness (Preservation Equipment Ltd, Diss, United Kingdom) treated with silicone to add strength and flexibility. Silicone mixture was prepared: 15 g Wacker silicone E4, 9 g Silicone oil AP 200 (Sigma-Aldrich, St. Louis, Missouri, United States) and $5.8 \mathrm{~g}$ Hexane. After carefully mixing, $1.5 \mathrm{mg}$ silicone mixture per $\mathrm{cm}^{2}$ was applied with a gloss paint roller. The siliconized membrane was left to polymerize overnight at room conditions. Final membrane thickness was measured with a micrometer. Membranes with a maximal thickness of $40 \mu \mathrm{m}$ were used for feeding. 
The feeding membrane was clamped in the feeding unit and $75 \mu \mathrm{l}$ methanol bovine hair extract was added to each well and left to dry for 30 minutes at room temperature in order to apply bovine scent to the siliconized side of the feeding membrane. Next, the unit was turned upside down and $R$. australis larvae were added to the wells (approximately 100 larvae per well). Netting was used to cover the plate and the lower plate was immediately mounted using the bolts to contain the larvae. The unit was then put upright, which stimulated contact between the serum and the larvae; as a result of their questing behavior larvae crawled up to the underside of the membrane.

The wells of the upper plate with the baudruche side of the feeding membrane at the bottom were disinfected using $70 \%$ ethanol and left to dry. Before serum was added to the in vitro feeding system, each $10 \mathrm{ml}$ of serum was supplemented with $5 \mu$ l gentamycin (Sigma-Aldrich, $10 \mathrm{mg} / \mathrm{ml}$ ). Six hundred $\mu \mathrm{l}$ serum was added to each well and replaced with fresh serum twice daily.

Serum samples were pre-warmed at $37{ }^{\circ} \mathrm{C}$ and subsequently added to the wells of the upper plate. The upper plate was sealed with an ELISA plate cover or Parafilm. The unit was placed in a $\mathrm{CO}_{2}$ incubator at $37{ }^{\circ} \mathrm{C}, 90 \%$ $\mathrm{RH}$ and $5 \% \mathrm{CO}_{2}$ (as a feeding stimulus) for $48 \mathrm{~h}$ to allow larvae to feed. Feeding was stopped by placing the feeding unit overnight at $-20{ }^{\circ} \mathrm{C}$ thus freezing the larvae. The percentage of larvae that were engorged (having an enlarged abdomen of at least 2 times the size of the dorsal shield) was determined visually using a stereomicroscope. The studies were performed blind in that the evaluator had no knowledge about the distribution of the test materials over the plate (see statistical evaluation below).

\section{Statistical evaluation}

In order to identify statistically significant results, samples were tested in six-fold. The samples were allocated to the feeding unit such that they were evenly distributed over the plate. This was done to prevent plate-position effects on feeding. The code was kept secret to the evaluator until after determination of the engorgement rate in each well. From the individual values, the average engorgement rate was calculated. Differences between engorgement rates obtained with different test materials were analyzed for statistical significance using one-way ANOVA (Graphpad Prism 5, Graphpad Software Inc.).

\section{Results}

\section{Production of antisera against rBm86 and rSUB in calves}

Friesian Holstein cattle were vaccinated with $\mathrm{rBm} 86$ or rSUB and the collected serum was tested for antigen specificity and antibody titers. Western blot analysis showed that bovine serum against rSUB or rBm86 both were specific to their respective recombinant antigens; the anti-rBm86 antiserum stained a protein at $\mathrm{Mw}$ $100 \mathrm{kDa}$, and the anti-rSUB antiserum stained a protein at $\mathrm{Mw} 25 \mathrm{kDa}$ and a protein at $\mathrm{Mw} 50 \mathrm{kDa}$, which could be a doublet of the Mw 25kDa protein (Fig. 1a, c). Specific antibody levels were subsequently quantified as endpoint titers (cut-off Bmin*2) for each specific antigen using a sandwich ELISA. The antibody endpoint titers were calculated as $2 \log$ values; generated anti-rBm86 serum had an endpoint titer of 17.0 (Fig. 1d). Vaccination with rSUB resulted in a $2 \log$ antibody end titer of 17.8 (Fig. 1b).

\section{Immunohistochemistry}

Immunochemistry was used to visualize which tissues were recognized by antibodies against $\mathrm{rBm} 86$ and $\mathrm{rSUB}$. Microscopic slides of whole unfed $R$. australis were incubated with anti-serum directed against each antigen. Antibodies against Bm86 were found to bind specifically to gut epithelium (Fig. 2). Staining of unfed $R$. australis larvae with antibodies directed against subolesin showed that anti-rSUB antibodies bound specifically to the acini of the salivary glands and to the epithelium of the rectal sac (Fig. 3a, c). Reactivity in the acini could be observed throughout the cytoplasm and the globular pattern of staining suggest that the antibodies might bind to small granules within the acini (Fig. 3b, d).

\section{Effect of anti-rBm86 and anti-rSUB bovine sera on in vitro tick feeding}

To determine the effect of the monospecific antisera against $\mathrm{rBm} 86$ and $\mathrm{rSUB}$ on engorgement, antisera were fed in vitro to 4-6 week-old $R$. australis larvae in six replicates. Of the larvae that were fed control (undiluted tick naïve bovine) serum, on average $44.3 \%$ had fed (data not shown). Feeding of larvae with undiluted antiserum against rSUB did not affect feeding as compared to the control serum (5\% reduction; Fig. 4). However, larvae that had been fed with undiluted antiserum against rBm86 exhibited reduced feeding (39\% reduction), but this difference did not reach statistical significance. Importantly, when feeding a mixture of equal volumes of serum raised against rBm86 and rSUB (hence each was tested at one time dilution) larval feeding was statistically significantly reduced by $62.7 \%(P=0.024)$ compared to control serum (Fig. 4).

In order to compare the effect of the two monospecific antisera with the mixture of the two sera at a similar dilution, the monospecific sera were one time diluted with tick naïve bovine serum. The mean feeding of larvae that were fed tick naïve bovine serum was $39.5 \%$ (control group). When larvae were fed either one-time diluted anti-rBm86 antiserum or one-time diluted anti-rSUB 

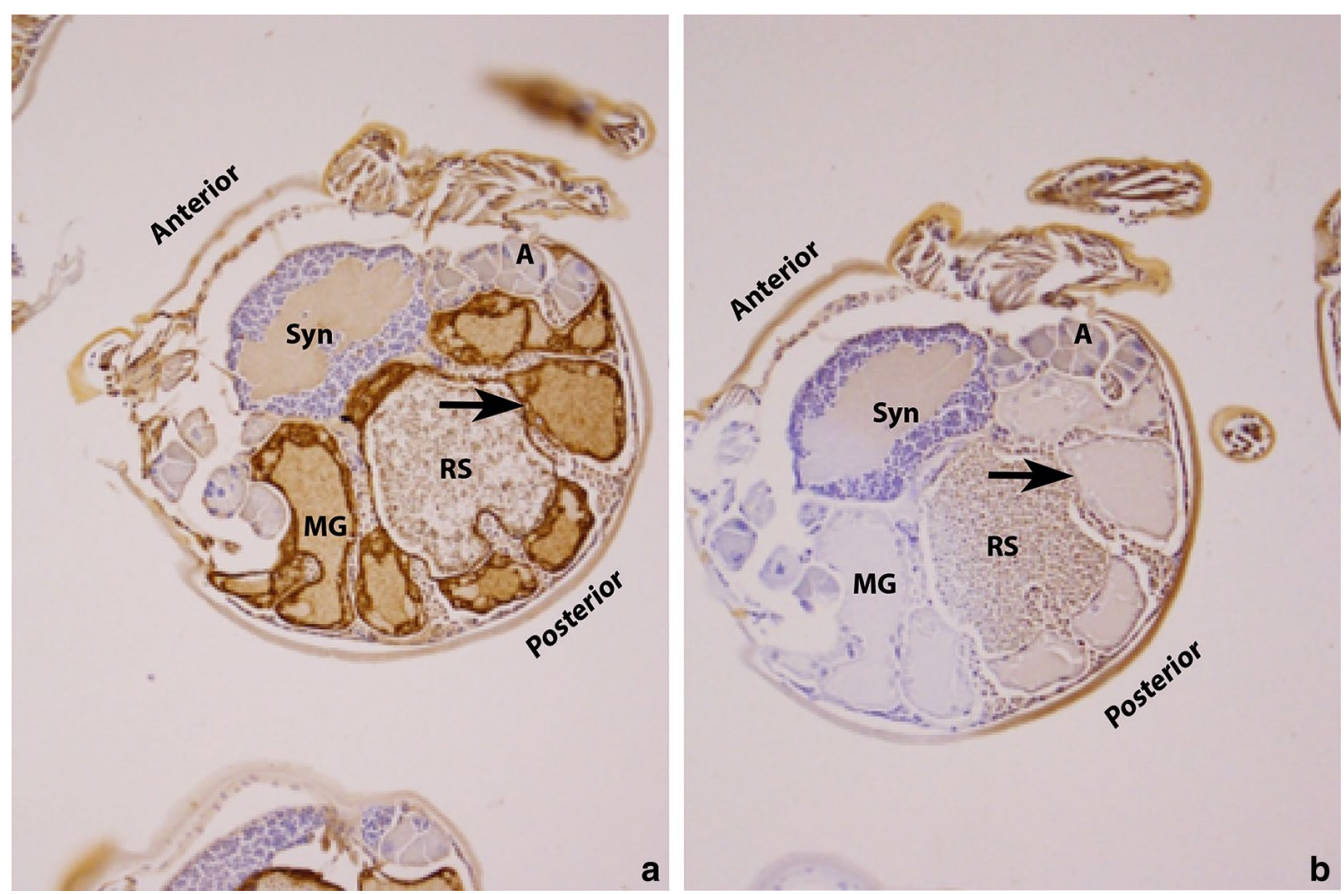

Fig. 2 Localization of Bm86 in unfed R. australis larvae. Cross sections (20x magnification) of larvae stained with: a rabbit anti-Bm86 serum (1:400 diluted) and $\mathbf{b}$ naïve rabbit serum (1:400 diluted). Sections were pre-treated with proteinase $\mathrm{K}$ for $30 \mathrm{~min}$ before antibody incubation. Antibodies bound specifically to midgut epithelium (indicated with arrows). Abbreviations: Syn, synganglion; MG, midgut; RS, rectal sac; A, acinus

antiserum, no inhibition of feeding was found (Fig. 5). However, when larvae were fed the mixture of antirBm86/anti-rSUB antiserum, feeding was reduced by $26.7 \%$. Although the observed effect was again highest with the mixture of the monospecific antisera, this difference did not reach statistical significance in this experiment $(P=0.095)$.

\section{Discussion}

In search for an improved anti-tick vaccine, the effectiveness of vaccine formulations that contain two or more tick antigens, which have shown partial protection when used as single-antigen vaccines, are being evaluated. The basis of such vaccine is the recombinant $R$. microplus midgut antigen $\mathrm{rBm} 86$ that is used in commercially available vaccines. Early experiments in immunized cattle have shown that protection is related to the antibody titer against $\mathrm{rBm} 86$ [36, 37], and in vitro feeding experiments with adult $R$. microplus showed that serum or purified immunoglobulins (Ig) from immunized cattle reduced the engorgement rate and oviposition in a high percentage of the ticks by damage of the midgut [38]. Similarly, vaccination of cattle with rSUB, a cytoplasmic and nuclear antigen from $R$. microplus ticks, induced partial protection that could be related to the level of anti-rSUB antibodies [39]. Before embarking on vaccination-challenge experiments in cattle to evaluate the efficacy of vaccination against both recombinant antigens, we studied the effect of anti-rBm86 and rSUB antibodies in vitro using a recently developed technique that allows feeding $R$. australis larvae with blood and/or serum [35]. Although the assay allows detecting statistically significant differences, variability can occur due to a number of factors such as tick age and condition, and batch differences of membranes and attractants. This can be overcome to some extent by increasing the number of replicates in the feeding assay. The results presented here show that the number of $R$. australis larvae able to feed is reduced when feeding undiluted anti-rBm86 antiserum from immunized cattle compared to tick naïve bovine serum, which is in line with earlier results [35]. When the Bm86 antiserum was diluted once with tick naive bovine serum the effect on tick feeding was lost. This is reminiscent of the work of Kemp and co-workers, who showed that an increase of anti-Bm86 IgG concentration to a concentration of twice that found in the original serum, increased the level of adult tick damage in vitro significantly [38]. Apparently, the effect of antiBm86 serum on feeding (and/or gut damage in vitro) is an almost a "yes" or "no" effect. In the same paper Kemp et al. show that antibodies can independently induce damage in feeding ticks. As antibodies block endocytosis 


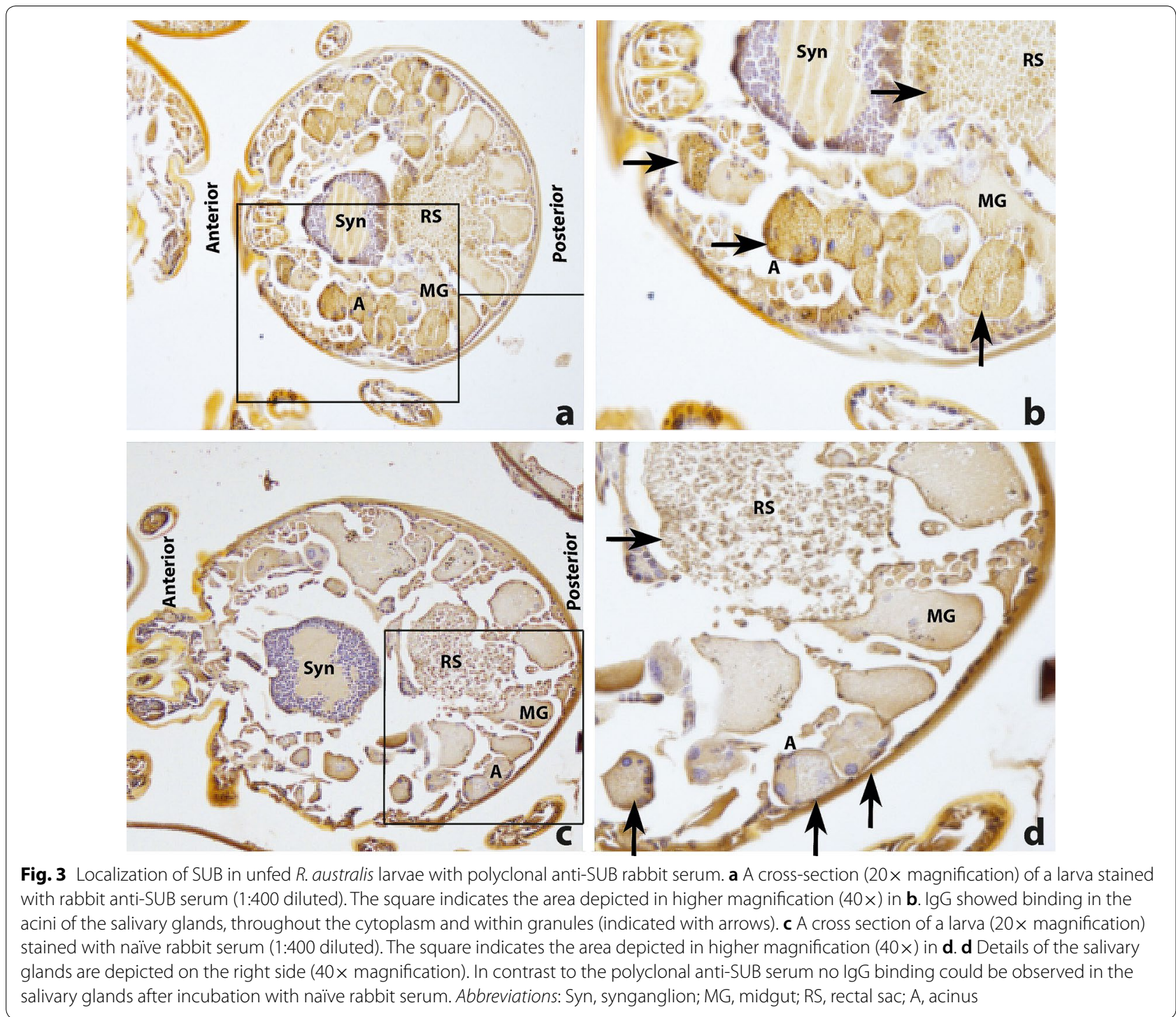

of fluorescein-labeled BSA by gut cells in vitro [5], one might hypothesize that blocking endocytosis adversely affects further engorgement of the larvae. Feeding larvae with undiluted anti-rSUB antiserum did not affect the feeding. Surprisingly, when larvae were fed anti-rBm86 serum that was 1:1 diluted with anti-rSUB antiserum, a $62.7 \%$ reduction of feeding was observed that was statistically significant $(P<0.05)$. This suggests that the action of anti-rBm86 antibodies allowed an additional and highly synergistic effect of anti-rSUB antibodies on tick feeding. It could be hypothesized that damage of gut epithelial cells, or lysis, exerted by anti-rBm86 antibodies is a prerequisite for antibodies against rSUB to exert their effect in ticks fed in vitro. Alternatively, the effect of antirBm86 could be catalyzed by the presence of anti-rSUB antibodies through an as yet unknown mechanism. Any explanation is at this point highly speculative.
In order to determine the localization of the proteins that were recognized by anti-rBm86 and anti-rSUB antibodies, immunohistochemistry was used. Due to the high background upon incubation with a conjugate against bovine Ig (data not shown) we used polyclonal rabbit sera that was raised against the two recombinant proteins instead of the bovine antisera. Antibodies against $\mathrm{rBm} 86$ were found to bind specifically to gut epithelium as has been reported previously, and did not stain the acini of the salivary glands [38, 40, 41]. In contrast, antibodies against rSUB reacted with some, but not all, of the acini of the salivary glands of unfed $R$. australis larvae. Reactivity in the acini was observed throughout the cytoplasm. This was unexpected as subolesin, which is a homologue of akirin, is thought to be an intranuclear protein $[14,15]$. Consequently, subolesin does not seem to be present only in the nucleus, or the observed 


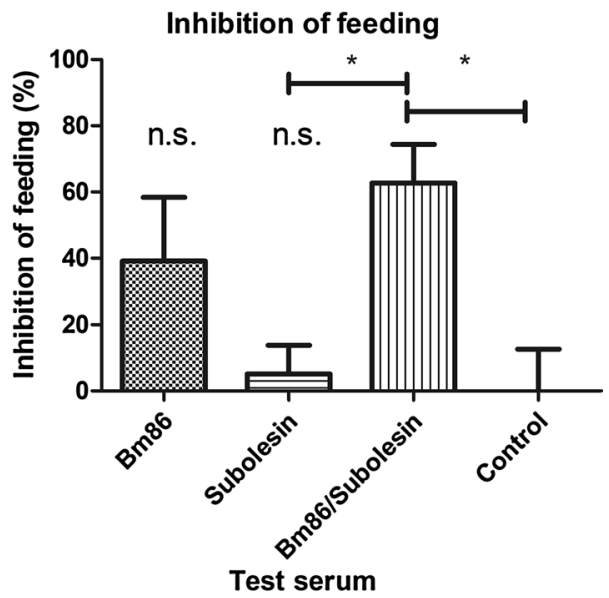

Fig. 4 The effect of monospecific antisera against tick antigens and a 1:1 combination of these antisera on $R$. australis larval feeding as compared to tick naïve bovine serum. Larvae of $R$. australis were fed in vitro on $600 \mu \mathrm{l}$ antisera against Bm86 or SUB or on $300 \mu \mathrm{l}$ anti-Bm86 bovine serum combined with $300 \mu l$ anti-SUB bovine serum. After $48 \mathrm{~h}$, ticks were visually scored for feeding. Bars represent the inhibition of feeding as compared to the control group, expressed as a percentage. Error bars represent the standard deviation. ${ }^{*} P<0.05$, n.s., not significant

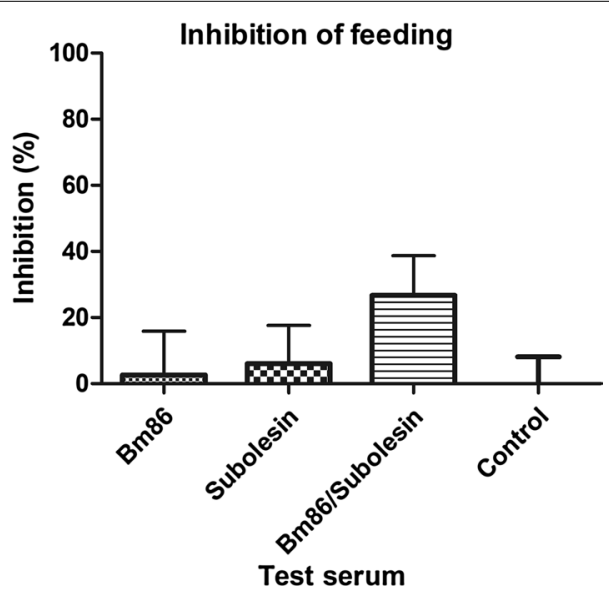

Fig. 5 The effect of one-time diluted monospecific antisera against tick antigens and a 1:1 mixture of these antisera on $R$. australis larval feeding as compared to tick naïve bovine serum. Larvae of $R$. australis were fed in vitro on $300 \mu$ lantisera against Bm86 or SUB diluted with $300 \mu \mathrm{l}$ naïve bovine serum. Again, $300 \mu \mathrm{l}$ anti-Bm86 bovine serum was combined with $300 \mu \mathrm{l}$ anti-SUB bovine serum to assess synergistic effects of both antigens. After $48 \mathrm{~h}$, ticks were visually scored for feeding. Bars represent the inhibition of feeding as compared to the control group, expressed as a percentage. Error bars represent the standard deviation

reaction outside the nucleus might be explained by crossreactivity of the anti-rSUB antibodies with an epitope on another protein. However, as the presence of SUB in the cytoplasm of cells in tick salivary glands was described previously for adult $R$. microplus [30], it is most likely that indeed SUB is also present in the cytoplasm of unfed larval salivary glands. Although the exact mechanism for the observed synergistic effect on larval feeding in vitro is unknown, it could be related to the different tick tissues targeted by each antibody; in $R$. australis larvae anti-rBm86 antibodies react with the gut epithelium and anti-rSUB antibodies react with the cytoplasm of acini in the salivary glands and with the epithelium of the rectal sac. While it is clear that biological variation can induce differences in effect sizes between in vitro assays, the presented in vitro reduction of the larval feeding gives high expectations for studying the efficacy of these antigens on $R$. australis infestation in vivo. Differences in effect sizes between in vitro assays, shows biological variation between tick batches. While feeding success for all control groups was similar, on average $40 \%$ of larvae attached and fed, expression levels of the target antigens might differ between ticks. For instance, for Bm86 it is known that expression levels are low in unfed larvae and shows relatively high variation compared to later life stages [42]. Differences in in vitro effect size due to biological variation in larvae would therefore likely to be less pronounced in the in vivo situation where all three life stages are involved. In vivo infestation experiments with $R$. australis and $R$. microplus on cattle span the entire life-cycle of the tick and therefore measures the summed effect of the tested vaccine on larval, nymphal and adult life stages and their respective molting periods. Since larvae are only the first stage of the life-cycle of $R$. australis and $R$. microplus and they only imbibe small amounts of blood compared to nymphal ticks and especially adult ticks, it might be very well possible that the combination of a vaccine targeting both $\mathrm{Bm} 86$ and subolesin will have a higher efficacy on these later stages.

\section{Conclusions}

To determine the potential protective effect of vaccines that contain a mixture of tick antigens, a 24-well in vitro feeding assay for $R$. australis larvae was used. It was found that feeding a combination of antisera rose against $\mathrm{rBm} 86$ and $\mathrm{rSUB}$ reduced the proportion of engorged larvae by $62.7 \%$ as compared to controls that received tick naïve bovine serum. As a result, a combined vaccine containing $\mathrm{rBm} 86$ and $\mathrm{rSUB}$ is appears to be a highly promising formula for further in vivo testing.

\section{Acknowledgments}

We like to thank Dr Heike Williams, Dr Hartmut Zoller, Mirjam Békefi and Bernd Jürgens from MSD Animal Health Innovation $\mathrm{GmbH}$, Research Antiparasitics for their support and the supply of ticks. 


\section{Authors' contributions}

TPMS initiated and supervised the study. JdIF and JWRH helped design the experimental set-ups and provided valuable input to shape the final manuscript. JJAT wrote the manuscript and performed membrane construction and feeding experiments. JAGMK, JvdC and JJAT participated in the cattle vaccinations, blood collection and ELISA's. HT performed immunohistochemistry. All authors read and approved the final manuscript.

\section{Funding}

The research was funded by MSD Animal Health. Aside from the authors, MSD Animal Health had no role in the study design, data collection and analysis or preparation of the manuscript.

\section{Availability of data and materials}

Data supporting the conclusions of this article are included within the article.

\section{Ethics approval and consent to participate}

Cattle vaccinations were approved by the animal ethical committee of MSD Animal Health.

\section{Consent for publication}

Not applicable.

\section{Competing interests}

TS, JT, JK and JC were employed by MSD Animal Health at the time of the described research.

\section{Author details}

${ }^{1}$ Center for Experimental and Molecular Medicine, Amsterdam Infection \& Immunity, Amsterdam UMC, Univ of Amsterdam, Meibergdreef 9, 1105AZ Amsterdam, The Netherlands. ${ }^{2}$ Mérieux NutriSciences, Pascalstraat 25, 6716 AZ Ede, The Netherlands. ${ }^{3}$ MSDAnimal Health, Wim de Körverstraat 35, 5830 AA Boxmeer, The Netherlands. ${ }^{4}$ Aduro Biotech Europe, Kloosterstraat 9, 1101 RX Boxmeer, The Netherlands. ${ }^{5}$ SaBio. Instituto de Investigación en Recursos Cinegéticos IREC (CSIC-UCLM-JCCM), 13005 Ciudad Real, Spain. ${ }^{6}$ Department of Veterinary Pathobiology, Center for Veterinary Health Sciences, Oklahoma State University, Stillwater, OK 74078, USA. ${ }^{7}$ ProtActivity, Sering 36, 5432 DD Cuijk, The Netherlands. ${ }^{8}$ Department of Veterinary Tropical Diseases, University of Pretoria, Onderstepoort 0110, South Africa.

Received: 24 March 2019 Accepted: 13 July 2019

Published online: 25 July 2019

\section{References}

1. Jonsson NN. The productivity effects of cattle tick (Boophilus microplus) infestation on cattle, with particular reference to Bos indicus cattle and their crosses. Vet Parasitol. 2006;137:1-10.

2. Estrada-Pena A, Venzal JM, Nava S, Mangold A, Guglielmone AA, Labruna MB, et al. Reinstatement of Rhipicephalus (Boophilus) australis (Acari: Ixodidae) with redescription of the adult and larval stages. J Med Entomol. 2012:49:794-802.

3. George JE, Pound JM, Davey RB. Chemical control of ticks on cattle and the resistance of these parasites to acaricides. Parasitology. 2004;129(Suppl. S1):S353-66.

4. Allen JR, Humphreys SJ. Immunisation of guinea pigs and cattle against ticks. Nature. 1979;280:491-3.

5. Willadsen P, Riding GA, McKenna RV, Kemp DH, Tellam RL, Nielsen JN, et al. Immunologic control of a parasitic arthropod. Identification of a protective antigen from Boophilus microplus. J Immunol. 1989;143:1346-51.

6. Fuente JDL, Almazán C, Canales M, Pérez de la Lastra JM, Kocan KM, Willadsen P. A ten-year review of commercial vaccine performance for control of tick infestations on cattle. Anim Health Res Revi. 2007:8:23-8.

7. Jonsson NN, Matschoss AL, Pepper P, Green PE, Albrecht MS, Hungerford J, et al. Evaluation of TickGARDPLUS, a novel vaccine against Boophilus microplus, in lactating Holstein-Friesian cows. Vet Parasitol. 2000;88:275-85.

8. Willadsen P. Anti-tick vaccines. Parasitology. 2004;129(Suppl.):S367-87.

9. de la Fuente J, Contreras M. Tick vaccines: current status and future directions. Expert Rev Vaccines. 2015;4:1367-76.
10. Rodríguez-Mallon A, Encinosa PE, Méndez-Pérez L, Bello Y, Rodríguez Fernández R, Garay H, et al. High efficacy of a 20 amino acid peptide of the acidic ribosomal protein P0 against the cattle tick, Rhipicephalus microplus. Ticks Tick Borne Dis. 2015;6:530-7.

11. Willadsen P, McKenna RV, Riding GA. Isolation from the cattle tick, Boophilus microplus, of antigenic material capable of eliciting a protective immunological response in the bovine host. Int J Parasitol. 1988;18:183-9.

12. Almazán C, Kocan KM, Bergman DK, Garcia-Garcia JC, Blouin EF, de la Fuente J. Characterization of genes transcribed in an Ixodes scapularis cell line that were identified by expression library immunization and analysis of expressed sequence tags. Gene Ther Mol Biol. 2003;7:43-59.

13. de la Fuente J, Almazán C, Blas-Machado U, Naranjo V, Mangold AJ, Blouin $E F$, et al. The tick protective antigen, 4D8, is a conserved protein involved in modulation of tick blood ingestion and reproduction. Vaccine. 2006;24:4082-95.

14. Galindo RC, Doncel-Pérez E, Zivkovic Z, Naranjo V, Gortazar C, Mangold $A J$, et al. Tick subolesin is an ortholog of the akirins described in insects and vertebrates. Dev Comp Immunol. 2009;33:612-7.

15. Goto A, Matsushita K, Gesellchen V, El Chamy L, Kuttenkeuler D, Takeuchi $\mathrm{O}$, et al. Akirins are highly conserved nuclear proteins required for NF-kappa B-dependent gene expression in drosophila and mice. Nat Immunol. 2008;9:97-104.

16. Almazán C, Kocan KM, Bergman DK, Garcia-Garcia JC, Blouin EF, de la Fuente J. Identification of protective antigens for the control of Ixodes scapularis infestations using cDNA expression library immunization. Vaccine. 2003;21:1492-501.

17. Almazán C, Lagunes R, Villar M, Canales M, Rosario-Cruz R, Jongejan F, et al. Identification and characterization of Rhipicephalus (Boophilus) microplus candidate protective antigens for the control of cattle tick infestations. Parasitol Res. 2010;106:471-9.

18. Nijhof AM, Taoufik A, de la Fuente J, Kocan KM, de Vries E, Jongejan F. Gene silencing of the tick protective antigens, Bm86, Bm91 and subolesin, in the one-host tick Boophilus microplus by RNA interference. Int J Parasitol. 2007;37:653-62.

19. Inokuma H, Kemp DH. Establishment of Boophilus microplus infected with Babesia bigemina by using in vitro tube feeding technique. J Vet Med Sci. 1998:60:509-12.

20. Lew-Tabor AE, Bruyeres AG, Zhang B, Rodriguez Valle M. Rhipicephalus (Boophilus) microplus tick in vitro feeding methods for functional (dsRNA) and vaccine candidate (antibody) screening. Ticks Tick Borne Dis. 2014;5:500-10.

21. Willadsen P, Kemp DH, McKenna RV. Bloodmeal ingestion and utilization as a component of host specificity in the tick, Boophilus microplus. Z Parasitenkd. 1984;70:415-20.

22. Kröber T, Guerin PM. An in vitro feeding assay to test acaricides for control of hard ticks. Pest Manag Sci. 2007;63:17-22.

23. Kröber T, Guerin PM. In vitro feeding assays for hard ticks. Trends Parasitol. 2007;23:445-9.

24. Kuhnert F, Diehl PA, Guerin PM. The life-cycle of the bont tick Amblyomma hebraeum in vitro. Int J Parasitol. 1995;25:887-96.

25. Musyoki JM, Osir EO, Kiara HK, Kokwaro ED. Comparative studies on the infectivity of Theileria parva in ticks fed in vitro and those fed on cattle. Exp Appl Acarol. 2004;32:51-67.

26. Doube BM, Kemp DH. The influence of temperature, relative humidity and host factors on the attachment and survival of Boophilus microplus (Canestrini) larvae to skin slices. Int J Parasitol. 1979;9:449-54.

27. Kemp DH, Koudstaal D, Roberts JA, Kerr JD. Feeding of Boophilus microplus larvae on a partially defined medium through thin slices of cattle skin. Parasitology. 1975;70:243.

28. Pierce AE, Pierce MH. A note on the cultivation of Boophilus microplus (Oanesteini, 1887) (Ixodidae: Acarina) on the embryonated hen egg. Aust Vet J. 1956;32:144-6.

29. Kocan KM, Yoshioka J, Sonenshine DE, de la Fuente J, Ceraul SM, Blouin EF, et al. Capillary tube feeding system for studying tick-pathogen interactions of Dermacentor variabilis (Acari: Ixodidae) and Anaplasma marginale (Rickettsiales: Anaplasmataceae). J Med Entomol. 2005;42:864-74.

30. Antunes S, Merino O, Mosqueda J, Moreno-Cid JA, Bell-Sakyi L, Fragkoudis R, et al. Tick capillary feeding for the study of proteins involved in tick-pathogen interactions as potential antigens for the control of tick infestation and pathogen infection. Parasit Vectors. 2014;7:42. 
31. Antunes S, Merino O, Lérias J, Domingues N, Mosqueda J, de la Fuente J, et al. Artificial feeding of Rhipicephalus microplus female ticks with anti calreticulin serum do not influence tick and Babesia bigemina acquisition. Ticks Tick Borne Dis. 2015;6:47-55.

32. Elvin CM, Kemp DH. Generic approaches to obtaining efficacious antigens from vector arthropods. Int J Parasitol. 1994;24:67-79.

33. Brossard M, Girardin P. Passive transfer of resistance in rabbits infested with adult Ixodes ricinus L.: humoral factors influence feeding and egg laying. Experientia. 1979;35:1395-7.

34. Roberts JA, Kerr JD. Boophilus microplus: passive transfer of resistance in cattle. J Parasitol. 1976;62:485-8.

35. Trentelman JJA, Kleuskens JAGM, van de Crommert J, Schetters TPM. A new method for in vitro feeding of Rhipicephalus australis (formerly Rhipicephalus microplus) larvae: a valuable tool for tick vaccine development. Parasit Vectors. 2017;10:153.

36. de la Fuente J, Rodriguez M, Redondo M, Montero C, Garcia-Garcia JC, Mendez $L$, et al. Field studies and cost-effectiveness analysis of vaccination with Gavac against the cattle tick Boophilus microplus. Vaccine. 1998;16:366-73

37. Willadsen P, Bird P, Cobon GS, Hungerford J. Commercialisation of a recombinant vaccine against Boophilus microplus. Parasitology. 1995;110(Suppl.):):S43-50.

38. Kemp DH, Pearson RD, Gough JM, Willadsen P. Vaccination against Boophilus microplus: Localization of antigens on tick gut cells and their interaction with the host immune system. Exp Appl Acarol. 1989;7:43-58.
39. Merino O, Almazán C, Canales M, Villar M, Moreno-Cid JA, Estrada-Peña A, et al. Control of Rhipicephalus (Boophilus) microplus infestations by the combination of subolesin vaccination and tick autocidal control after subolesin gene knockdown in ticks fed on cattle. Vaccine. 2011;29:2248-54

40. Kopp N, Diaz D, Amacker M, Odongo DO, Beier K, Nitsch C, et al. Identification of a synthetic peptide inducing cross-reactive antibodies binding to Rhipicephalus (Boophilus) decoloratus, Rhipicephalus (Boophilus) microplus, Hyalomma anatolicum anatolicum and Rhipicephalus appendiculatus BM86 homologues. Vaccine. 2009;28:261-9.

41. Odongo D, Kamau L, Skilton R, Mwaura S, Nitsch C, Musoke A, et al. Vaccination of cattle with TickGARD induces cross-reactive antibodies binding to conserved linear peptides of Bm86 homologues in Boophilus decoloratus. Vaccine. 2007;25:1287-96.

42. Nijhof AM, Balk JA, Postigo M, Jongejan F. Selection of reference genes for quantitative RT-PCR studies in Rhipicephalus (Boophilus) microplus and Rhipicephalus appendiculatus ticks and determination of the expression profile of Bm86. BMC Mol Biol. 2009;10:112.

\section{Publisher's Note}

Springer Nature remains neutral with regard to jurisdictional claims in published maps and institutional affiliations.
Ready to submit your research? Choose BMC and benefit from:

- fast, convenient online submission

- thorough peer review by experienced researchers in your field

- rapid publication on acceptance

- support for research data, including large and complex data types

- gold Open Access which fosters wider collaboration and increased citations

- maximum visibility for your research: over 100M website views per year

At BMC, research is always in progress.

Learn more biomedcentral.com/submissions 